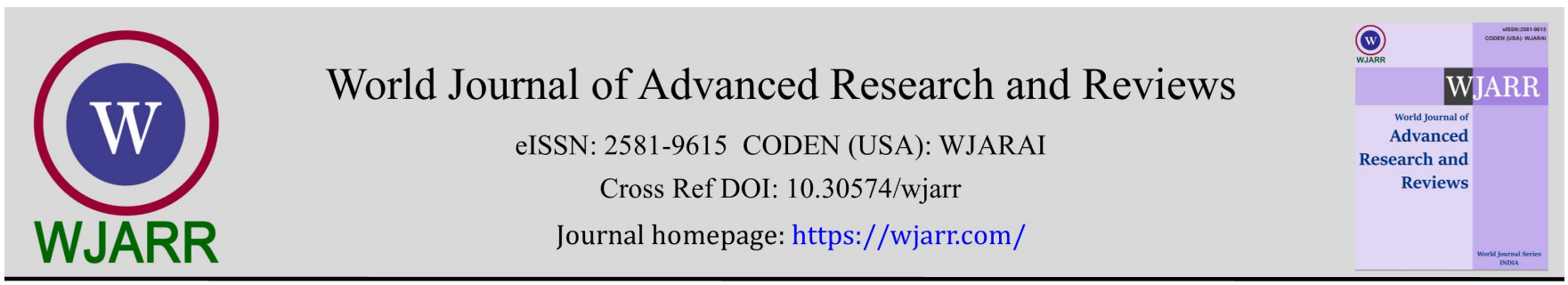

(REVIEW ARTICLE)

\title{
Review: Radio Electric Asymmetric Conveyer (REAC) as adjuvant therapy of mental disorder
}

\author{
Putri Aliya Ahadini ${ }^{1}$, Muhamad Bagus Wira Utama ${ }^{1}$, Adhyatma Ismu Reihan ${ }^{1}$ and Reny I'tishom ${ }^{2, *}$ \\ ${ }^{1}$ Medical Program, Faculty of Medicine, Universitas Airlangga, Surabaya, Indonesia. \\ 2 Department of Biomedical Science, Faculty of Medicine, Universitas Airlangga, Surabaya, Indonesia.
}

World Journal of Advanced Research and Reviews, 2022, 13(01), 280-284

Publication history: Received on 07 December 2021; revised on 09 January 2022; accepted on 11 January 2022

Article DOI: https://doi.org/10.30574/wjarr.2022.13.1.0030

\begin{abstract}
Mental disorders are one of the health disorders that contribute to high rates of disability and mortality worldwide. The current therapeutic modalities used to treat mental disorders are medical and psychological approaches, but it becomes problematic in some conditions, such as drug-resistant mental disorders. Radio Electric Asymmetric Conveyer (REAC) technology can be used as an alternative to overcome this problem. This technology uses radio waves which are guaranteed to be non-invasive and do not cause side effects. This technology enables neuromodulation effects by maximizing cell polarity and optimizing endogenous bioelectric activity. Of course, the REAC's mechanism as a neuromodulator and being a non-invasive technology is safe to use. It allows REAC to be used as an adjuvant therapy to reduce symptoms of several mental disorders such as depression, anxiety, bipolar disorder, phobias, and stress.
\end{abstract}

Keywords: REAC; Brain stimulation; Efficacy; Mental disorder

\section{Introduction}

Mental disorders are an essential health problem today. Mental disorders consist of various symptoms generally characterized by abnormal thoughts, emotions, behaviors, and relationships with other people. Based on the DSM-V, the areas of mental disorders covered include organic disorders, mental and behavioral disorders due to the use of psychoactive substances, schizophrenia, schizotypal and delusional disorders, mood (affective) disorders, neurotic disorders, behavioral syndromes related to physiological disorders, and physical factors, personality and behavioral disorders in adults, mental retardation, disorders of psychological development, behavioral and emotional disorders with onset in childhood and adolescence, and unspecified mental disorders [1].

People with mental disorders experience much higher rates of disability and death. People with major depression and schizophrenia are $40 \%$ to $60 \%$ more likely to die prematurely than the general population [2]. Depression accounts for $4.3 \%$ of the global disease burden and is one of the most significant causes of disability worldwide [2]. Based on this, it can be concluded that mental disorders can cause disability and mortality, impacting a country's economy.

In recent years, neurostimulation techniques have been developed because of ineffective pharmacological treatments in drug-resistant mental disorders. Radio Electric Asymmetric Conveyer (REAC) technology is designed to optimize endogenous bioelectric activity. REAC works on the modulation of cell polarity and the intracellular environment, where the efficacy of REAC is as optimization of cell polarity. Endogenous bioelectrical mechanisms enable cellular functions, such as cell migration, proliferation, differentiation, regeneration, and nerve transmission [3,4,5]. The neuromodulatory effect of REAC on synaptic and neuronal activity has been observed simultaneously from two brain regions (thalamus

\footnotetext{
* Corresponding author: Reny I'tishom

Departement of Biomedical Science, Faculty of Medicine, Universitas Airlangga, Surabaya, Indonesia.

Copyright (C) 2022 Author(s) retain the copyright of this article. This article is published under the terms of the Creative Commons Attribution Liscense 4.0.
} 
and somatosensory cortex). The REAC trial on chronic pain rats showed a significant effect, suggesting the primary influence of REAC at the cellular level on the brain's functional network architecture [6]. The somatosensory cortex plays a role in emotion regulation. Some mental disorders are associated with abnormal emotion regulation (major depression, bipolar disorder, schizophrenia, post-traumatic stress, anxiety and panic, phobias, obesity, obsessivecompulsive disorder), as structural and functional changes are found. somatosensory cortex (delete this) [7]. Based on how it works and the sharing of (delete this) benefits, it is necessary to conduct an assessment to determine the effect of this therapeutic modality in humans. This review article aims to examine the effectiveness of REAC as adjuvant therapy for mental health disorders. This technology is non-invasive, so it is not harmful to the recipient. If its (it's) efficacy is proven, it can be used as one of the new adjuvant therapy options applied to patients with mental disorders.

\section{Results and discussion}

\subsection{Radio Electric Asymmetric Conveyer}

REAC is a technology for neuromodulation and biomodulation. This technology produces low-intensity radio-electric emission, which is $5.8 \mathrm{GHz}$. Radio-electric emissions interact with all structures that contain an electrical charge, such as the human body. REAC is used to optimize cell polarity to trigger good intraneuronal transmission [8]. Optimization of endogenous bioelectric activity also occurs because REAC emits an environmental radio-electric field directly into the patient's body through the Asymmetric Conveyer Probe (ACP) device [9].

REAC has advantages such as being non-invasive, not causing hyper- or hypo-polarization phenomena, having no depth limit, no side effects and being long-lasting. There are two types of REAC, namely REAC Neuro Postural Optimization Treatment (REAC-NPO) and REAC Neuro Psycho-Physical Optimization Treatment (REAC-NPPO). Both are distinguished by how they are used and their purpose. REAC-NPO is a neuromodulatory therapy used to increase efficiency and function throughout the brain. This technology can optimize neurotransmission in encephalitis to treat neurodegenerative diseases, such as Parkinson's and Alzheimer's. This therapy is done with a single treatment for 250 milliseconds in the auricle area or earlobe. REAC-NPPO is a technology aimed at optimizing the allostatic response to stressors. It causes a decrease in dysfunctional adaptive attitudes, which are symptoms and pathologies in several neuropsychological diseases such as depression, anxiety, and stress. One cycle of REAC-NPPO neuromodulation treatment consisted of 18 sessions. Each session lasts about 3 seconds, and the treatment is given to a specific area, i.e., the auricle or earlobe $[8,9]$.

\subsection{Efficacy of REAC as Adjuvant Therapy for Mental Disorders}

\subsubsection{REAC Efficacy on Depression}

Several studies of REAC in both animals and humans in helping treat depression have been tested. Studies in rat models of parkinsonism induced by the intoxicant 1-methyl-4-phenyl-1,2,3,6-tetrahydropyridine (MPTP) in vivo found that REAC tissue optimization treatment specific for neuro-regenerative purposes (REAC TO-RGN-N) attenuated the inflammatory features evoked by nigrostriatal damage in MPTP-induced mice, decreased levels of pro-inflammatory molecules, and increased anti-inflammatory mediators. This suggests that REAC TO-RGN-N modulates the proinflammatory response and reduces neuronal damage in MPTP-induced parkinsonism [10]. Increased inflammatory activity affects the peripheral and central nervous systems, which is attributed to its role in developing depression [11]. REAC therapy in depressed patients showed promising results; a significant decrease in depressive symptoms was obtained. This indicates that the use of REAC can help reduce the symptoms of depression $[8,12]$. Even the use of REAC in patients experiencing depression related to the COVID-19 pandemic showed similar results, namely a decrease in the degree of depression of the patients [9].

\subsubsection{REAC Efficacy on Anxiety}

The role of REAC as a therapeutic option for anxiety management has been observed in several experimental studies. Pinheiro et al., 2020 conducted research on people who experience mental or psychological disorders due to the COVID19 pandemic conditions. REAC NPO and NPPO CB therapy were performed on participants, and evaluation based on DASS-21 before and after treatment was performed to measure anxiety symptoms. After undergoing a series of REAC NPO and CB NPPO therapy for three weeks, it was found that there was a decrease in the severity of anxiety symptoms based on the mean score of the DASS-21 test. Before undergoing REAC therapy, the average severity of anxiety symptoms in participants was moderate; after treatment, the anxiety symptoms decreased to normal [9].

Research done by Rinaldi (2019), assessed the efficacy of specific neuromodulation therapy given using Radio Electric Asymmetric Conveyer (REAC) technology as therapy in overcoming symptoms of depression, anxiety, and stress. The 
subjects of this study were people who had depression, anxiety, or stress symptoms based on the DASS-42 test. The research subjects were given two different treatments, the first group would undergo REAC NPO and REAC NPPO for three months, and the second group, as the control group, did not receive any action. As a result, the group receiving REAC NPO and REAC NPPO therapy experienced decreased severity of symptoms of depression, anxiety, and stress based on the DASS-42 test score. While in the control group, there was no significant change [8].

\subsubsection{REAC Efficacy on Panic Disorder}

Previous studies have investigated the effect of administering REAC on patients who have agoraphobia. Agoraphobia is a condition where a person experiences excessive panic symptoms when in situations that are considered highly embarrassing. This condition is considered to be the most severe complication of panic disorder [13]. An agoraphobic person tends to avoid places or situations that could induce a panic attack. If they are caught in these situations, they will experience extreme discomfort and the conviction of an imminent panic attack [14]. The treatment of agoraphobia is done by giving pharmacological therapy with serotonin reuptake inhibitors and norepinephrine reuptake inhibitors. However, in most cases, these approaches cannot solve the problem [15].

This study was conducted on 23 patients ( 3 males and 20 females) who had been diagnosed with agoraphobia according to DSM IV criteria [16]. The age range of the research object is 34-41 years. The agoraphobia patient was given two 18session cycles of non-invasive brain stimulation with REAC-NPPO. The symptom scale was assessed at each REAC session with the Agoraphobia Scale [17]. The experimental results concluded that the AS value decreased significantly as the patient was given brain stimulation therapy. The majority of the patients felt globally better after the first cycle of REAC-NPPO therapy. After the second cycle of treatment, the improvement from baseline was more evident [18]. This indicates the potential of the REAC to treat complex clinical situations such as agoraphobia, which is typically resistant to pharmacologic treatments. The rapid therapeutic effect, appearing in just a few months, highlights necessary time and economic advantages, even compared to novel cognitive-behavioral treatment strategies.

\subsubsection{REAC Efficacy on Bipolar Disorder}

Bipolar disorder is a mental disorder that causes drastic changes in mood in sufferers and repeated episodes with symptoms of mania and depression [19]. The pathophysiology of bipolar disorder is still unknown. Failure to regulate the neurotransmitter noradrenaline is known to cause depressive episodes [19]. Symptoms of mood disorders can also be affected by dysregulation in dopamine and serotonin [19]. Current therapy is limited to pharmacological and psychosocial approaches. REAC can be an alternative supporting therapy in this disorder based on its pathophysiology.

REAC-lithium given to four groups of patients who have bipolar disorder showed good effects [20]. The groups were differentiated based on the follow-up time, namely 72 months, 48 months, 36 months, and 18 months [20]. In all four groups there was a reduction in the incidence of both mania and depressive episodes [20]. This is possible because of the REAC activity that allows the potentiation of neurobiological activity.

\subsubsection{REAC Efficacy on Social Phobia/Social Anxiety Disorder (SAD)}

In 2011, an experimental study was conducted to compare the effects of REAC-NPO therapy with the results of sertraline administration in patients with Social Anxiety Disorder. This was a 6-month, open-label, naturalistic study. This study divides the research object into two groups based on the type of therapy. The first group received 2 cycles $\mathrm{x} 18$ sessions of REAC-NPPO, while the second group received sertraline therapy at a $125.0 \pm 15.5 \mathrm{mg} /$ day once a day. After evaluating the symptoms of Social Anxiety Disorder on the object, it was concluded that REAC-NPPO had a better effect in reducing Social Anxiety Disorder symptoms and that NPPO-REAC was globally statistically superior to standard dosages of sertraline on selected primary efficacy criteria, CGI (Clinical Global Improvement) improvement, and total LSAS score for fear and avoidance [21].

\subsubsection{REAC Efficacy on Stress}

In Rinaldi et al. [22] it was found that REAC can be an alternative therapy for stress-related disorders. Based on the randomized control study, 150 people in the group treated with REAC found a significant reduction in Psychological Stress Measure (PSM) scores, compared to 50 people in the control group who received a placebo. This shows that REAC can reduce symptoms and subjective views of sufferers regarding the stress they are experiencing.

Based on the DSM-V, there are several categories of stress-related disorders, including Post-Traumatic Stress Disorder (PTSD), Acute Stress Disorder (ASD), Adjustment Disorder, Reactive Attachment Disorder (RAD), Disinhibited Social Engagement Disorder, as well as specific and non-specific, and specific stress-related disorder. Stress-related disorders increase the risk of sufferers experiencing life-threatening infections, mainly at a young age [23]. Stress, particularly in 
childhood, has also been identified as a risk factor for many psychiatric disorders, including recurrent manic symptoms in bipolar disorder and schizophrenia [24,25].

Based on a study conducted by Renaldi et al. [8] for stress clusters, 71 participants were at an average level, 32 at moderate, 36 at mild, 34 at severe, and nine at very severe. After three months of treatment, the stress cluster found 140 participants at normal levels, 16 at moderate levels, 15 at mild levels, seven at severe levels, and four at very severe levels. Based on this research, there was a significant decrease. Based on this study, a comparison between the treatment and control groups proved that REAC treatment could improve quality of life. In the second DASS test, conducted approximately 3 months after treatment, it was found that the treated participants achieved significantly lower mean scores in all three groups, depression, anxiety, and stress [8].

Based on the research of Pinheiro et al. [9] for the stress group, 77 (49.68\%) participants were found to be positive: 28 males (36.36\%) and 49 females (63.64\%). The overall mean age of this group was $50.93 \pm 13.50$ years. At T0, the mean stress score was 22.61, corresponding to moderate severity. At T5, the mean stress score was 10.14 , corresponding to average severity. In 64 (83.12\%) participants, REAC treatment reduced stress symptoms [9].

REAC can be a therapeutic option for stress in several ways. Among them, REAC can increase cell polarity, leading to increased intraneuronal transmission, thereby inhibiting the pathophysiology of stress [12]. Inhibited mechanisms include dysregulation of dopamine in the Noradrenaline Transporter (NET) and the dysregulation of 5-HT1A and 5HT1B receptors. In addition, REAC also optimizes the response to stressors so that it can cause a decrease in dysfunctional adaptive behavior [15]. In addition, REAC is also known to be an effective instrument for improving responses to allostatic conditions and environmental stressors [22].

\section{Conclusion}

The role of REAC in the treatment of mental disorders in the future is crucial. Several potential REACs that have been tested have shown promising results in treating mental disorders such as depression, anxiety, bipolar disorder, phobias, panic disorder, and stress. This can provide an option to the community that there is a non-invasive therapy who has a good effect in curing various mental disorders. It is necessary to conduct further studies on the usefulness of REAC in mental disorders in humans so that more data obtained will assist the development of REAC and the application of REAC to mental disorders.

\section{Compliance with ethical standards}

\section{Acknowledgments}

All authors have contributed to this study equally. We thank all the lecturers at the Faculty of Medicine, Universitas Airlangga who have guided and supported us while making this manuscript to completion.

\section{Disclosure of conflict of interest}

There is no conflict of interest between the authors.

\section{References}

[1] American Psychiatric Association. American Psychiatric Association: Diagnostic and Statistical Manual of Mental Disorders. 2013; 5.

[2] World Health Organization. Mental Health Action Plant 2013-2020 [Internet]. Apps.who.int. 2013 [cited 3 January 2022].

[3] Maioli M, Rinaldi S, Santaniello S, Castagna A, Pigliaru G, Delitala A, et al. Anti-senescence efficacy of radio-electric asymmetric conveyer technology. AGE. 2013; 36(1): 9-20.

[4] Rinaldi S, Meloni MA, Galleri G, Maioli M, Pigliaru G, Cugia G, et al. Radio Electric Asymmetric Conveyer (REAC) technology to obviate loss of T cell responsiveness under simulated microgravity. PLOS ONE. 2018; 13(7).

[5] Maioli M, Rinaldi S, Santaniello S, Castagna A, Pigliaru G, Delitala A, et al. Radioelectric asymmetric conveyed fields and human adipose-derived stem cells obtained with a nonenzymatic method and device: A novel approach to multipotency. Cell Transplantation. 2014; 23(12):1489-500. 
[6] Rinaldi S, CalzÃ L, Giardino L, Biella GE, Zippo AG, Fontani V. Radio Electric Asymmetric Conveyer: A novel neuromodulation technology in alzheimer and other Neurodegenerative Diseases. Frontiers in Psychiatry. 2015; 6.

[7] Kropf E, Syan SK, Minuzzi L, Frey BN. From anatomy to function: The role of the somatosensory cortex in emotional regulation. Brazilian Journal of Psychiatry. 2019; 41(3): 261-9.

[8] Rinaldi A, Rinaldi C, Coelho Pereira JA, Lotti Margotti M, Bittencourt MN, Barcessat AR, et al. radio electric asymmetric Conveyer neuromodulation in depression, anxiety, and stress. Neuropsychiatric Disease and Treatment. 2019; 15: 469-80.

[9] Pinheiro Barcessat AR, Nolli Bittencourt M, Duarte Ferreira L, de Souza Neri E, Coelho Pereira JA, Bechelli F, et al. REAC cervicobrachial neuromodulation treatment of depression, anxiety, and stress during the COVID-19 pandemic. Psychology Research and Behavior Management. 2020; 13: 929-37.

[10] Panaro MA, Aloisi A, Nicolardi G, Lofrumento DD, De Nuccio F, La Pesa V, et al. Radio Electric Asymmetric Conveyer technology modulates neuroinflammation in a mouse model of neurodegeneration. Neuroscience Bulletin. 2017; 34(2): 270-82.

[11] Lee C-H, Giuliani F. The role of inflammation in depression and fatigue. Frontiers in Immunology. $2019 ; 10$.

[12] Mannu P, Rinaldi S, Fontani V, Castagna A. Radio electric asymmetric brain stimulation in the treatment of behavioral and psychiatric symptoms in alzheimer disease. Clinical Interventions in Aging. 2011; 207.

[13] Grant BF, Hasin DS, Stinson FS, Dawson DA, Goldstein RB, Smith S, et al. The epidemiology of DSM-IV Panic Disorder and agoraphobia in the United States. The Journal of Clinical Psychiatry. 2006; 67(03): 363-74.

[14] Cassano GB, Michelini S, Shear MK, Coli E, Maser JD, Frank E. The panic-agoraphobic spectrum: A descriptive approach to the assessment and treatment of subtle symptoms. American Journal of Psychiatry. 1997; 154(6): 27-38.

[15] Galassi F, Quercioli S, Charismas D, Niccolai V, Barciulli E. Cognitive-behavioral group treatment for panic disorder with agoraphobia. Journal of Clinical Psychology. 2007; 63(4): 409-16.

[16] Schmidt NB, Salas D, Bernert R, Schatschneider C. Diagnosing agoraphobia in the context of panic disorder: Examining the effect of DSM-IV criteria on diagnostic decision-making. Behaviour Research and Therapy. 2005; 43(9): 1219-29.

[17] Öst L-G. The Agoraphobia Scale: An Evaluation of its reliability and validity. Behaviour Research and Therapy. 1990; 28(4): 323-9.

[18] Mannu P, Rinaldi S, Fontani V, Castagna A, Lotti M. Noninvasive brain stimulation by radioelectric asymmetric conveyor in the treatment of agoraphobia: Open-label, Naturalistic study. Patient Preference and Adherence. $2011 ; 575$.

[19] Agrim J. Bipolar Disorder: Diagnosis, Pathophysiology and Therapy. EC Neurology. 2020; 12(8): 136-149.

[20] Piero M, Rinaldi S, Fontani V, Castagna A. Long-term treatment of bipolar disorder with a radioelectric asymmetric conveyor. Neuropsychiatric Disease and Treatment. 2011; 373.

[21] Fontani V, Rinaldi S, Mannu P, Castagna A. Social anxiety disorder: Radio electric asymmetric conveyor brain stimulation versus sertraline. Patient Preference and Adherence. 2011; 581.

[22] Rinaldi S, Fontani V, Aravagli L, Mannu P. Psychometric evaluation of a radio electric auricular treatment for stress related disorders: a double-blinded, placebo-controlled controlled pilot study. Health and Quality of Life Outcomes. 2010; 8(1): 31.

[23] Song H, Fall K, Fang F, Erlendsdóttir H, Lu D, Mataix-Cols D et al. Stress related disorders and subsequent risk of life threatening infections: population based sibling controlled cohort study. BMJ. 2019: 15784.

[24] Smoller J. The Genetics of Stress-Related Disorders: PTSD, Depression, and Anxiety Disorders. Neuropsychopharmacology. 2015; 41(1): 297-319.

[25] Gilman S, Ni M, Dunn E, Breslau J, McLaughlin K, Smoller J, et al. Contributions of the social environment to firstonset and recurrent mania. Molecular Psychiatry. 2014; 20(3): 329-336. 